\title{
NIVEL DE ACTIVIDAD FISICA, SEDENTARISMO Y VARIABLES ANTROPOMETRICAS EN FUNCIONARIOS PUBLICOS
}

\author{
Carolina Alemán R. y Walter Salazar R. \\ Escuela de Educación Física y Deportes \\ Universidad de Costa Rica, San José, Costa Rica \\ E-mail:caleman03@costarricense.cr
}

\begin{abstract}
Resumen
Alemán-Ramírez, C. y Salazar-Rojas, W. (2006). Nivel de actividad física, sedentarismo y variables antropométricas en funcionarios públicos. Revista de Ciencias del Ejercicio y la Salud, 4(1), 1-12. El propósito de la investigación fue conocer la cantidad de actividad física que los funcionarios realizan durante la semana. Para ello participaron 84 personas con una edad promedio de 30.69 años, todos funcionarios del Programa de Atención Integral de Salud convenio UCR-CCSS; a quienes se les midió, el IMC, la circunferencia de la cintura y la cantidad de actividad física que realizaban por semana por medio del cuestionario de actividades físicas de Paffenbarger. Mediante análisis de varianza de una vía se determinaron diferencias significativas entre la circunferencia de la cintura de las mujeres y los hombres. No se encontró diferencia significativa en cuanto al nivel de sedentarismo entre hombres y mujeres. En cuanto al IMC, un $23 \%$ del total de la muestra presentó sobrepeso y un $14 \%$ obesidad. Estos datos indican porcentajes muy altos en factores de riesgo para la salud física de estos funcionarios, a pesar de ser adultos jóvenes, lo que lleva a la conclusión de que es imprescindible la intervención con programas que modifiquen estos factores de riesgo, como la práctica regular de actividad física, por medio de planes de acción diseñados de acuerdo a las posibilidades económicas, a las facilidades de acceso a lugares apropiados para la práctica física, o bien la educación de los funcionarios por parte de profesionales en el campo, para que puedan involucrar dentro de sus quehaceres diarios un estilo de vida más activo. PALABRAS CLAVES: actividad física, sedentarismo, gasto calórico, índice de masa corporal, circunferencia de la cintura:
\end{abstract}

\section{INTRODUCCIÓN}

En Costa Rica las enfermedades cardiovasculares son una de las principales causas de muerte. Esta situación se viene presentando principalmente desde la década de los 70, así mismo es notorio que la edad en que aparecen estas enfermedades va en descenso año tras año (Ministerio de Salud, 1994); este hecho parece demostrar que no se están realizando las medidas que detengan o minimicen esto, como por ejemplo campañas fuertes de prevención, sino por el contrario es evidente el aumento de algunos de los factores que causan dichas enfermedades.

Se han estudiado las causas o variables a las cuales se les podría atribuir el aumento de esta situación y se han encontrado algunos factores de riesgo como por ejemplo la edad, el género, antecedentes familiares, obesidad, fumado, sedentarismo, entre otros. Algunos poseen más prevalencia que otros, pero la suma de estos podría aumentar el riesgo (Hernández, 2000; y Esquivel, Suárez, Calzada, Sandí y Ureña, 2002).

Hernández (2000), encontró que la obesidad y el sedentarismo eran los factores de riesgo con más prevalecencia en una población obrera industrial de la provincia de Cartago, ya que el $87.23 \%$ eran sedentarios y el $53.19 \%$ presentaron obesidad. Esto muestra un ejemplo de dos factores de riesgo que podrían estar presentándose con mucha incidencia en la población costarricense.

Uno de estos factores es la obesidad; esta es una enfermedad crónica de causas multifactoriales, que se caracteriza por un exceso de tejido graso, que se manifiesta por un peso inadecuado generalmente como el 
resultado de un exceso de la ingesta calórica, es decir por factores conductuales $\mathrm{o}$ ambientales, o bien que puede desarrollarse por factores genéticos y hormonales, entre otros (Serrano, Field y Prieto, 1994).

A pesar de que existen métodos más precisos para conocer si una persona es o no obesa como por ejemplo resonancia magnética, dexa, impedancia magnética; su diagnóstico generalmente se hace por medio del índice de masa corporal (IMC), el cual, a pesar de que no toma en cuenta la composición corporal de las personas, es un método sencillo y práctico que ha sido utilizado en muchas investigaciones epidemiológicas (Bar- Or, 2003) y también se valora a diario en clínicas, hospitales o centros de salud. El IMC se calcula de la siguiente manera: peso $(\mathrm{kg})$ dividido entre la estatura (m) al cuadrado, es decir es la relación del peso con respecto a la estatura; lo recomendable es que las personas posean un IMC entre 18.5 y 24.9 ; cuando es menor de 18.5 se considera bajo, cuando se encuentra entre 24.9 y 30 se clasifica con sobrepeso y cuando es superior de 30 se clasifica como obesa (Pollock y Wilmore, 1990).

Otro método que se ha empleado es determinar la circunferencia de la cintura, porque se ha encontrado una fuerte relación entre enfermedades cardiovasculares y la cantidad de grasa a nivel abdominal. Cuando las mujeres presentan $\geq 88 \mathrm{~cm}$ y los hombres $\geq 102 \mathrm{~cm}$ en la circunferencia de la cintura se encuentran con una circunferencia grande por lo que es conveniente disminuir el porcentaje de grasa y / o el peso corporal ya que se presenta un alto riesgo de padecer enfermedades cardiovasculares (Jakicic y cols, 2001).

Al igual que en países industrializados, la obesidad en Costa Rica va en ascenso en todos los grupos de edad (Hernández, 2000). Es un problema muy grave de salud pública porque aumenta el riesgo de presentar algunos tipos de cáncer, problemas de fertilidad, hipertensión arterial, dislipidemia, enfermedades coronarias, y hasta muerte súbita. En el año de 1982 un $31.5 \%$ de la población costarricense presentaba sobrepeso y obesidad, para el año de 1996 esta cifra aumentó al $49.53 \%$, y estudios más recientes pero en lugares específicos como por ejemplo en Tibás en 1996, Desamparados en 1999 y Cartago en 2001, este porcentaje se encontró entre el $55-60 \%$ de las poblaciones estudiadas (Sáenz, 2004).

Como anteriormente se mencionó otro factor de riesgo es el sedentarismo, además es una de las causas que conduce a la obesidad (Powell, 1987). Por ejemplo en Estados Unidos entre el $60-80 \%$ de la población se clasifica como sedentaria y esto se correlaciona con el porcentaje de sobrepeso y obesidad (Sánchez, García, Landabaso y Martínez, 1998),

Un indicador del nivel de sedentarismo de los costarricenses podría ser el aumento de la obesidad, sin embargo no se cuenta con una base que indique el ascenso o descenso del mismo, ni tampoco se conoce una cifra que revele el nivel de actividad o de inactividad física que poseen los ticos en la actualidad. El Ministerio de Salud (1994), señala un estudio que se llevó a cabo por el IAFA, el cuál reveló que sólo el $3 \%$ de la población analizada realizaba actividad física de manera frecuente; sin embargo este estudio es muy general porque no especifica género, edad, ni tampoco señala la población de la que se tomaron los datos.

Para el Colegio Americano de Medicina del Deporte, una persona sedentaria es aquella que no realiza actividades físicas durante 30 minutos al día, al menos tres veces a la semana. Ellos han definido recomendaciones en cuanto a la regularidad, frecuencia y duración de la misma; se debe de realizar entre 20-60 minutos continuos o intermitentes, con un mínimo de 3 veces a la semana y a una intensidad del $55-65 \%$ hasta el $90 \%$ de la frecuencia cardiaca (Scott, Morrow, Jackson y Dunn, 2000).

Por otro lado Jakicic y cols. (2001), así como también Haapanen, Miilunpalo Vuori, Oja y Pasanen (1996), mencionan que las personas que no generan un gasto calórico superior de 2000 kilocalorías por semana en 
actividades físicas, son sedentarias. Estos autores toman esta recomendación porque se ha encontrado que es donde se obtienen los mayores beneficios a nivel cardiovascular.

Alemán y Salazar (2003), investigaron los niveles de actividad física en estudiantes universitarios; se tomó la recomendación de que el gasto calórico semanal en actividades físicas debe ser superior de 2000 kilocalorías. Los resultados mostraron que el $53 \%$ de los estudiantes eran sedentarios, además los participantes justificaron su inactividad por la falta de tiempo y mencionaron que no conocen cuánta actividad física deben de realizar. Con este estudio, una vez más se demostró que adultos jóvenes, ya poseen factores de riesgo para presentar algún tipo de patología.

En el 2004 Alemán y Salazar, analizaron los niveles de actividades físicas pero esta vez en funcionarios de una universidad pública. La investigación mostró que estas personas sí eran físicamente activas, porque mantenían gastos calóricos superiores a las 2000 kilocalorías (Jakicic y cols, 2001). Algunas de las razones por las que se da esta situación podría ser la ubicación de la universidad que facilita el acceso a gimnasios, también podría ser por la flexibilidad en el horario de trabajo o bien como lo mencionan Sternfeld, Ainsworth y Quesenberry (1999), los individuos con estudios universitarios se preocupan por su salud, por lo que realizan regularmente actividad física.

No se conocen con certeza todas las razones por las cuales las personas no realizan actividades físicas vigorosas con regularidad; podría ser por los avances tecnológicos, que han mejorado las condiciones laborales, como el uso de internet, así como también los medios de transporte; por nuevas formas en el uso del tiempo libre como los juegos electrónicos, la televisión, e inclusive podría ser por la publicidad, ya que se le vende a las personas la idea de mejorar su apariencia y estado de salud sin ningún esfuerzo físico, como por ejemplo las vendas frías, los batidos mágicos, o "fajas reductoras".
Aunque sí se tiene conocimiento acerca de todos los beneficios que brinda la práctica regular de actividad física, tanto a nivel físico como mental (Horvat, French y Henschen, 1986), falta que las personas hagan conciencia y la lleven a la práctica. Es tarea de todos los profesionales del campo de la salud, como nutricionistas, médicos de las diferentes especialidades, fisioterapistas, y educadores físicos, educar en estos aspectos a las personas con las que se relacionan en las respectivas ocupaciones.

Además los individuos pueden involucrar dentro de su quehacer diario ciertas actividades con más movimiento como subir gradas o caminar al supermercado. Un ejemplo de este estilo de vida lo poseen los Amish, quienes son una comunidad agrícola que no cuenta con las facilidades modernas; por lo que tienen que realizar una serie de actividades físicas en donde se observan gastos energéticos significativos. Un estudio realizado con ellos, encontró que este estilo de vida contribuye a que estas personas posean bajos índices de obesidad (4\%) y sobrepeso (26\%) (Bassett, Schneider, y Huntington, 2004). Esto contrasta mucho con los datos obtenidos en Estados Unidos en donde el $30.9 \%$ de las personas presentan obesidad y el $64.5 \%$ poseen sobrepeso (Flegal, Carroll, Ogden, y Jonson, 2002), y también con Canadá donde el $14,9 \%$ de la población es obesa y el $50,7 \%$ tienen sobrepeso (Katzmarzyk, 2002). Por tanto, es importante enfatizar que un estilo de vida activo disminuye la posibilidad de presentar sobrepeso u obesidad.

Por otro lado, se ha encontrado que la participación en la práctica regular de actividad física ha estado influenciada por diversos factores como el género, la edad, el nivel educativo, el ingreso económico, las destrezas individuales y la psicología (Stewart, Trost, Owen, Sallis y Brown 2002). Yang, Telama, Leino, Viikari (1999), en su investigación determinan que los niños y los adolescentes son más activos que los adultos; pero conforme aumenta la edad, disminuye el número de actividades que requieren un 
mayor gasto energético. Para estos autores uno de los aspectos que influye en el cambio de actividad física son los cambios sociales que brinda la vida con el transcurso del tiempo.

Pratt, Macera, y Blanton (1999), encontraron resultados similares; ellos estudiaron la actividad física en jóvenes y en adultos y obtuvieron que el $63,8 \%$ de los estudiantes de colegio eran físicamente activos (más de 20 minutos, 3 o más veces a la semana), mientras que sólo el $28 \%$ de los adultos eran activos.

Otro aspecto relacionado con la disminución de la actividad física es el género. En el estudio de Yang, Telama, Leino, Viikari (1999), se determinó que las mujeres generan un gasto calórico inferior al de los hombres. Sternfeld, Ainsworth y Quesenberry (1999), encontraron en su investigación que las mujeres no realizan ejercicio, y esto podría ser porque muchas mujeres se dedican a labores del hogar, por lo que no disponen de tiempo libre, para realizar dichas actividades. Sin embargo también se observó que tanto los hombres como las mujeres profesionales, sí realizaron con frecuencia actividades físicas que involucren un gasto energético representativo.

Para determinar el nivel de actividad física, se debe calcular el gasto calórico que genera dicha actividad, tomando en cuenta que es un componente con mucha variación porque incluye actividades de la vida diaria, como por ejemplo las actividades deportivas, recreativas, laborales, entre otras. No obstante existen otros componentes del gasto energético total del cuerpo como lo son la tasa metabólica basal, que representa el $50 \%-70 \%$, y el efecto térmico de los alimentos que genera un gasto del $7 \%$ al $10 \%$ (Kriska, 1997).

Ahora bien una forma de medir el nivel de actividad física es por medio de cuestionarios, porque se pueden aplicar a una cantidad considerable de personas y además el costo económico de estudios que los utilicen, es más accesible.
El cuestionario de Paffenbarger Physical Activity Questionnaire (Sallis, Haskell y Wood, 1996), se ha utilizado en varias investigaciones y los datos que se recopilan de él, es decir las kilocalorías que gastan las personas, se han correlacionado significativamente con diversas variables, por ejemplo Ainswort, Leon, Richardson, Jacobs y Paffenbarger (1993) encontraron una correlación de 0.60 , entre las kilocalorías y el consumo máximo de oxígeno, por otro lado Washburn, Smith, Goldfield, y McKinlay (1991) obtuvieron una correlación de 0.14 entre las kilocalorías y el colesterol HDL y de 0.13 con el IMC.

En Costa Rica se necesita conocer si los estilos de vida modernos y los avances tecnológicos y científicos, están llevando a sus habitantes a ser personas sedentarias por lo que no sólo podrían tener riesgo de presentar problemas de salud sino también a que exista un incremento de la demanda y en los gastos en el sistema de salud pública.

Con el propósito de observar y determinar el gasto energético, la circunferencia de la cintura y el índice de masa corporal, se ha desarrollado la presente investigación, para así conocer estas variables $y$ poder compararlas con recomendaciones establecidas; también para determinar si existen ciertos factores de riesgo para la salud física en funcionarios públicos.

Por otro el lado brindar información en cuanto al porcentaje de sedentarismo en estos funcionarios, podría contribuir a la formación de programas que promuevan la práctica frecuente de actividad física.

\section{METODOLOGIA}

\section{Sujetos}

Se contó con 84 participantes en el estudio. Todos eran funcionarios del Programa de Atención Integral de Salud, convenio UCR- CCSS. Se realizaron mediciones en las oficinas del nivel central, en las coordinaciones de las áreas de salud de 
San Juan, de Montes de Oca, de Curribadat y en los Ebais de Vargas Araya, San Diego, y de Curridabat. Se contó con la participación de 12 médicos, 8 ATAP (técnicos en atención primaria) 2 redes, 17 secretarias, 12 misceláneas, 17 enfermeras, 3 registros médicos, 3 trabajadoras sociales, 3 choferes, 2 sociólogos, 2 nutricionistas, 1 mensajero y 2 auxiliares de especialidad. El total de mujeres fue de 57 y el total de hombres fue de 27. La edad promedio fue de $30,69 \pm 7,44$ años.

\section{Instrumentos}

Para determinar el nivel de actividades físicas se utilizó el cuestionario llamado "Actividades Físicas Habituales Paffenbarger" (Sallis, Haskell, y Wood, 1996). Este instrumento de medición está conformado por los siguientes apartados: kilómetros que la persona camina durante el día, las gradas que sube, la frecuencia, tipo y duración de las actividades deportivas y /o recreativas que realizan las personas. Con los datos obtenidos se calcularon las calorías relativas que gastan las personas durante la semana. También los participantes desglosaron las horas que dedican a la ejecución de actividades durante un día entre semana y en un día de fin de semana, clasificando las actividades en vigorosas (cavar el jardín, carpintería pesada, deportes fuertes, correr, danza aeróbica, nado, caminata rápida, bicicleta en colinas), actividades moderadas (trabajar en casa, deporte liviano, caminata regular, pintar, bailar, carpintería liviana), actividades livianas (trabajar en la oficina, manejar carro, permanecer de pie con poco movimiento) y actividades que se realizan estando sentado (comer, leer, trabajo de escritorio, ver televisión, escuchar radio).

Así mismo se realizaron mediciones antropométricas como el peso, la estatura, la circunferencia de la cintura. Para conocer el peso de las personas se utilizó una báscula electrónica marca Tanita HD-309, con la que se obtuvo el peso en kilogramos.
Para la medición de la circunferencia de la cintura se utilizó una cinta métrica. Se midió en la parte más estrecha de la cintura entre la última costilla y la cresta ilíaca.

\section{Procedimiento}

Los datos se recolectaron en los respectivos lugares de trabajo de los sujetos. Estos participantes laboraban en el programa de atención integral de salud convenio UCRCCSS, en las coordinaciones de salud de San Juan, de Montes de Oca, de Curribadat y en los Ebais de Vargas Araya, San Diego, y de Curridabat.

Las mediciones se llevaron a cabo en una oficina designada para dicho fin, con lo que se logró la individualidad y privacidad de cada una de las personas. A cada participante se le explicó el objetivo del estudio, y su participación en el mismo fue voluntaria.

Cuando la persona concluía el cuestionario se verificaba que estuviera completo, cada uno tardó aproximadamente 10 minutos en responder. Las mediciones se llevaron a cabo sin ninguna dificultad porque los participantes estaban vestidos con ropa holgada y liviana, estas mediciones tuvieron una duración aproximada de 15 minutos.

\section{Análisis estadístico}

Por medio del programa SPSS 8,0 se obtuvo la estadística descriptiva y la inferencial, que consistió en 15 análisis de varianza (ANOVA) de una vía (variable independiente sexo) para el peso, IMC, cintura, gasto calórico, gasto calórico relativo, dormir entre semana, dormir fin de semana, actividades vigorosas entre semana, actividades vigorosas fin de semana, actividades moderadas entre semana, actividades moderadas fin de semana, actividades livianas entre semana, actividades livianas fin de semana, actividades sentados entre semana, actividades sentados fin de semana. También se realizaron 4 ANOVA de 2 vías (sexo por ocupación) para el IMC, la 
circunferencia de la cintura, gasto calórico total y el absoluto.

\section{RESULTADOS}

En la Tabla 1 se puede apreciar la estadística descriptiva (promedios y desviaciones estándar) para cada una de las variables según el género y según el valor total.

Por otra parte, en la Tabla 2 se presentan los resultados de las pruebas $\mathrm{F}$ del análisis de varianza entre las mujeres y los hombres en cada una de las variables. Como se puede observar, hubo diferencias significativas en las variables: peso, cintura, actividad vigorosa entre semana, actividad vigorosa fin de semana, y actividad moderada fin de semana $(\mathrm{p}<0,05)$.

En la Tabla 3 se presentan los resultados de las pruebas $\mathrm{F}$ del análisis de varianza de dos vías para grupos independiente $2 \times 9$ (género por ocupación), en las siguientes variables: IMC, circunferencia de la cintura, gasto calórico total, y gasto calórico relativo. Como se puede apreciar, solamente hubo diferencias significativas en la circunferencia de cintura entre hombres y mujeres.

Tabla 3. Resultados de las pruebas $F$ en cada una de las variables analizadas en el ANOVA de 2 vías

\begin{tabular}{lllc}
\hline Variables & Sexo & Ocupación & $\begin{array}{c}\text { Sexo x } \\
\text { ocupación }\end{array}$ \\
\cline { 2 - 4 } $\begin{array}{l}\text { Gasto calórico } \\
\text { total }\end{array}$ & 1,92 & 0,87 & 1,49 \\
$\begin{array}{l}\text { Gasto calórico } \\
\text { relativo }\end{array}$ & 1,52 & 0,001 & 1,56 \\
$\begin{array}{l}\text { I.MC } \\
\begin{array}{l}\text { Circunferencia } \\
\text { de cintura }\end{array}\end{array}$ & 0,49 & 0,934 & 0,642 \\
\hline$* \mathrm{p}<0,05$ & $6,219 *$ & 0,49 & 1 \\
\hline
\end{tabular}

A continuación en la Figura 1, donde se muestra el gasto calórico en las mujeres y en los hombres de acuerdo a su ocupación.
Figura 1. Gasto Calórico según género y ocupación

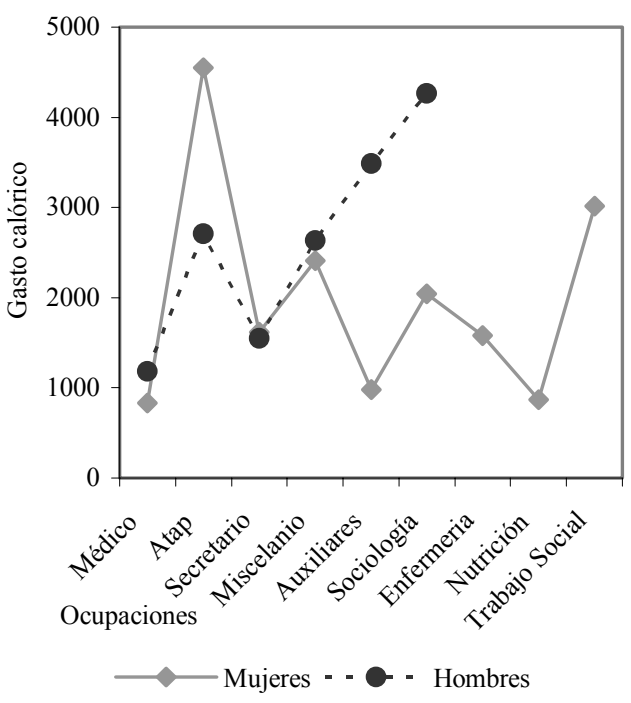

Se observa que las ATAP, los(as) sociólogos(as), los(as) misceláneos(as) y las trabajadoras sociales, presentan un gasto calórico semanal de 2000 kilocalorías. Mientras que los que poseen un menor gasto calórico son los auxiliares, los médicos y nutricionistas. En la Figura 2 se muestra el gasto calórico por géneros. No se encuentran diferencias en cuanto al gasto calórico entre los hombres y las mujeres. Las mujeres no gastan más de 2000 kilocalorías, por otro lado los hombres, sí llegan a un gasto calórico superior al de 2000 kilocalorías.

\section{Figura 2. Gasto calórico por género}

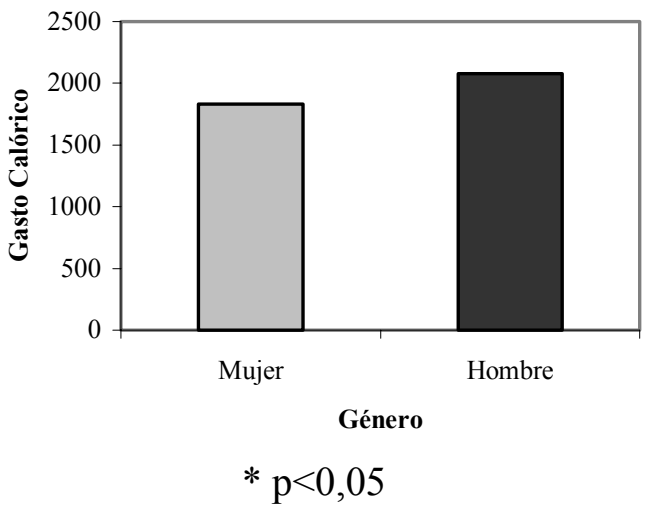


Tabla 1.Estadística descriptiva de cada una de las variables analizadas

\begin{tabular}{lccc}
\hline & Promedio \pm & Promedio \pm & Promedio \pm \\
DS mujeres & DS Hombres & $30,69 \pm 7,44$ \\
\hline Edad (años) & $31,28 \pm 7,60$ & $29,59 \pm 7,45$ & $1,61 \pm 0,20$ \\
Estatura (metros) & $1,55 \pm 0,21$ & $1,71 \pm 0,36$ & $66,36 \pm 13,71$ \\
PePeso (kilogramos) & $61,87 \pm 11,68$ & $75,81 \pm 13,72$ & $24,94 \pm 4,28$ \\
Índice de masa corporal & $24,8 \pm 4,68$ & $25,22 \pm 3,34$ & $82,49 \pm 10,87$ \\
Cintura (cm) & $80 \pm 10,94$ & $87,75 \pm 8,76$ & $1912,4 \pm 1467,52$ \\
Gasto energético (kcal) & $1834,98 \pm 1499,73$ & $2076,4 \pm 1411,7$ & $7,24 \pm 1,40$ \\
Horas duermen/ semana & $7,15 \pm 1,58$ & $7,43 \pm 0,85$ & $8,50 \pm 2,19$ \\
Horas duermen fin de semana & $8,5 \pm 2,41$ & $8,3 \pm 1,57$ & $0,96 \pm 1,4$ \\
Actividades vigorosas entre semana & $0,7 \pm 1,01$ & $1,55 \pm 2,02$ & $1,60 \pm 1,66$ \\
Actividades vigorosas fin de semana & $1,31 \pm 1,41$ & $2,43 \pm 2,02$ & $3,74 \pm 3,27$ \\
Actividades moderadas / semana & $4,04 \pm 3,65$ & $3,11 \pm 2,10$ & $3,96 \pm 3,18$ \\
Actividades moderadas fin de sema & $4,44 \pm 3,53$ & $2,87 \pm 1,81$ & $7,46 \pm 3,48$ \\
Actividades livianas entre semana & $7,48 \pm 3,18$ & $7,42 \pm 3,39$ & $5,19 \pm 4,36$ \\
Actividades livianas fin de semana & $5,08 \pm 4,43$ & $5,47 \pm 4,29$ & $5,15 \pm 2,96$ \\
Actividades sentados entre semana & $4,9 \pm 2,98$ & $5,65 \pm 2,91$ & \\
\hline
\end{tabular}

Tabla 2. Resultados de las pruebas $\mathbf{F}$ entre las mujeres y los hombres en las variables analizadas

\begin{tabular}{lc}
\hline \multicolumn{1}{c}{ Variables } & Sexo \\
\hline Peso & $24,20^{*}$ \\
IMC & 0,17 \\
Cintura & $10,38^{*}$ \\
Gasto calórico & 0,19 \\
Gasto calórico relativo & 0,318 \\
Dormir entre semana & 0,64 \\
Dormir fin de semana & 0,22 \\
Act. Vigorosas entre semana & $4,45^{*}$ \\
Act. Vigorosas fin de semana & $5,47^{*}$ \\
Act. Moderadas entre semana & 1,08 \\
Act. Moderadas fin de semana & $4,23^{*}$ \\
Act. Livianas entre semana & 0,005 \\
Act. Livianas fin de semana & 0,12 \\
Act. Sentados entre semana & 0,12 \\
Act. Sentados fin de semana ** & \\
\hline
\end{tabular}

$* \mathrm{p}<0,05 * *$ Esta variable no se respondió.

En la Figura 3, se observa que no existe diferencia significativa entre hombres $\mathrm{y}$ mujeres en cuanto al gasto calórico relativo, es decir al gasto calórico por peso corporal

Figura 3. Gasto calórico por kilogramo de peso corporal

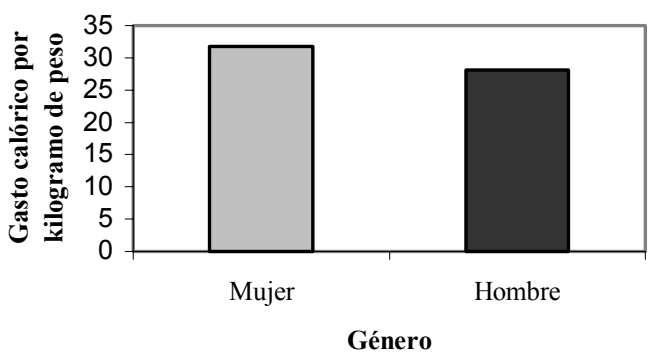

También se obtuvo el porcentaje de sedentarismo y de actividad física de acuerdo al género, estos datos se presentan en la Tabla 4, se observa que el porcentaje de sedentarismo es bastante alto tanto en las mujeres como en los hombres.

Tabla 4. Porcentaje de personas Activas y Sedentarias

\begin{tabular}{lcc}
\hline & Activos & Sedentarios \\
\cline { 2 - 3 } Mujeres & $40 \%$ & $60 \%$ \\
Hombres & $33 \%$ & $67 \%$
\end{tabular}


Como se puede apreciar en la Figura 4 , no se encontraron diferencias significativas en cuanto al I.M.C entre mujeres y hombres. Las mujeres presentan un promedio en el I.M.C de 24,8, es decir se encuentran en el límite superior de normopeso (Pollock y Wilmore, 1990), y están en el límite inferior para presentar sobrepeso (IMC, entre 25-30). Por otro lado los hombres sí poseen un IMC superior de 25, es decir tienen sobrepeso.

Figura 4. Índice de masa corporal (I.M.C)

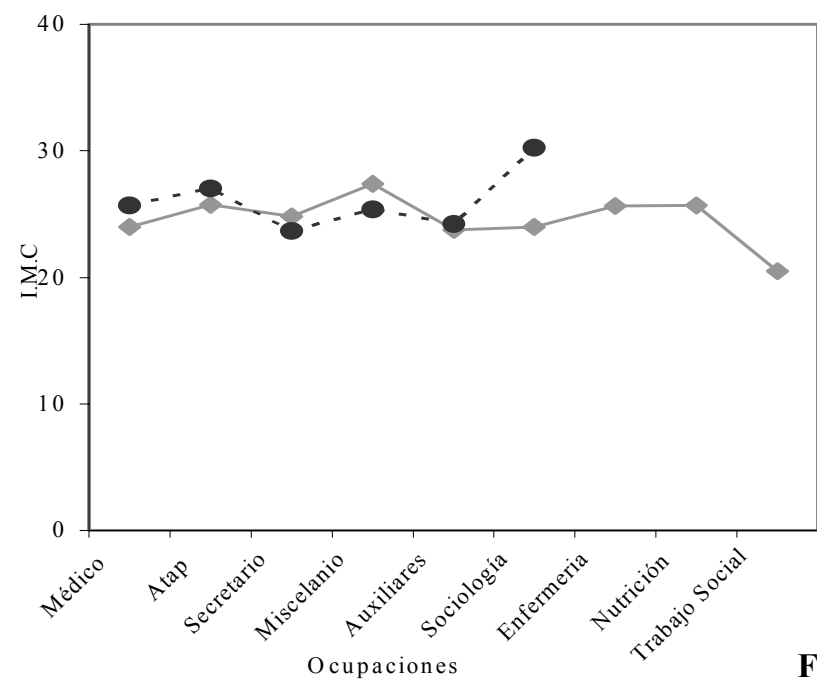

Figura 5. Porcentaje de la muetra en cada parámetro recomendado

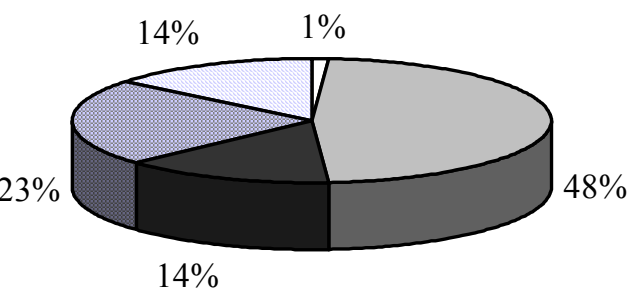

口Menor de 18,5 (1\%)

口Entre 18,5- 24 (48\%)

Entre 24- 24,9 (14\%)

口Entre 24,9-30 (23\%)

पMayor de 30 (14\%)
Por otra parte, en la Figura 5 se presenta el porcentaje de la muestra en cada uno de los parámetros recomendados para el IMC. Se observa que el $1 \%$, posee un IMC inferior de 18,5 , el $62 \%$ de la muestra posee un IMC normal es decir entre 18,5 y 24,9 , sin embargo este porcentaje se muestra de forma separada para señalar que el $14 \%$ del mismo se encuentra entre 24 y 24,9 en el límite superior, el $23 \%$ de la muestra posee sobrepeso, y el $14 \%$ posee obesidad.

En la Figura 6 se observan los promedios de la circunferencia de la cintura. Los hombres poseen más circunferencia que las mujeres. Las mujeres tienen un promedio de $80 \mathrm{~cm}$ y los hombres de $87 \mathrm{~cm}$, ambos se encuentran dentro de las recomendaciones hechas por el Colegio Americano de Medicina Deportiva (ACSM, 2006).
Figura 6. Circunferencia de la cintura (cm).

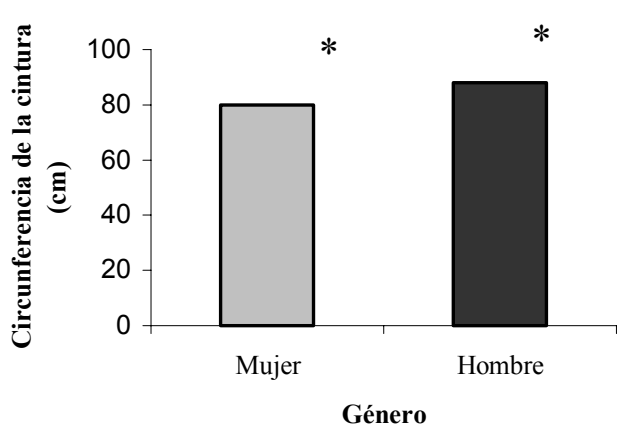

*: estadísticamente diferentes entre ellos $(\mathrm{p}<0.05$

En la figura 7, se pueden observar los porcentajes de la muestra correspondientes a cada parámetro recomendado para las mujeres (debe ser inferior de $88 \mathrm{~cm}$ ). 
Figura 6. Porcentaje de la muestra femenina en el rango de cintura recomendado

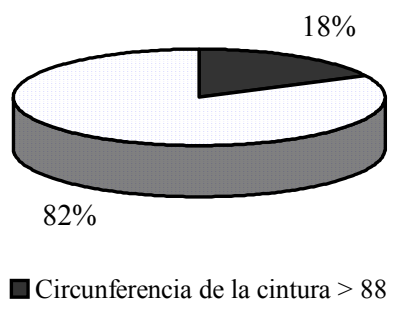

$\square$ Circunferencia de la cintura $<88$

En la Figura 8.se presenta el porcentaje de la muestra, en cuanto a las recomendaciones de la circunferencia de la cintura los hombres (debe ser inferior de 102 $\mathrm{cm})$.

Figura 8. Porcentaje de la muestra masculina en el rango de cintura recomendado

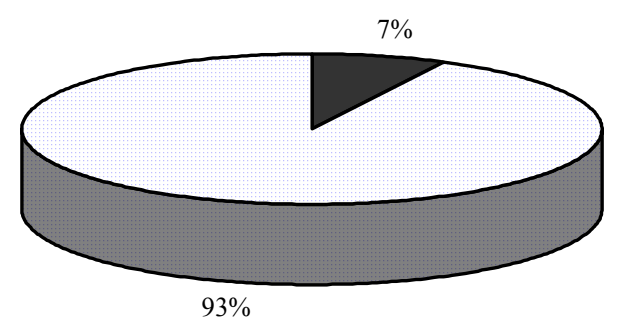

Circunferencia de la cintura $>102$

$\square$ Circunferencia de la cintura $<102$

En la figura 9 se presenta las horas que los participantes dedican a realizar actividades vigorosas, moderadas, livianas y sentados durante la semana y el fin de semana. Las mujeres sí realizan más actividades moderadas durante el fin de semana que los hombres. En las otras actividades no se encuentran diferencias significativas.
Figura 9. Promedio de horas que dedican las mujeres y los hombres en realizar actividades vigorosas, moderadas, livianas y sentados en el día

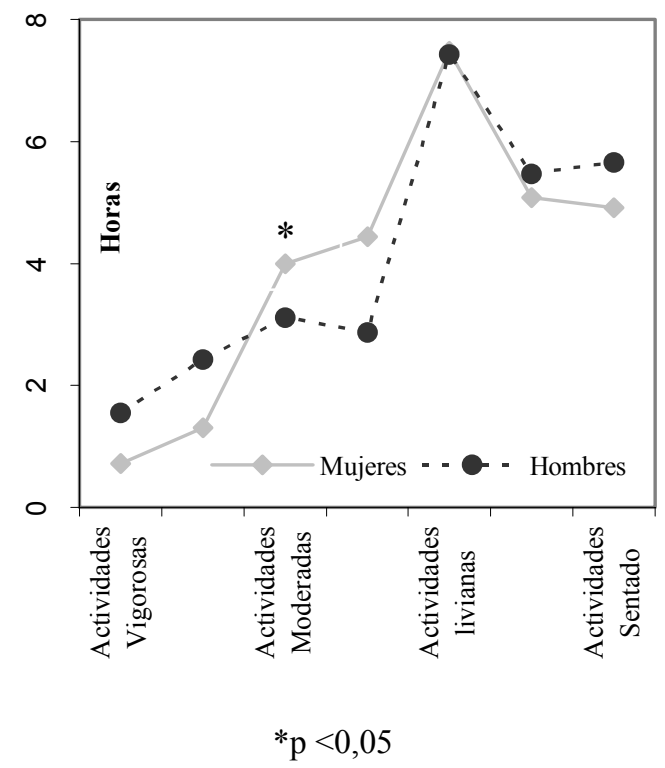

Los resultados obtenidos muestran los niveles de actividad física, el gasto calórico relativo y los promedios del IMC la relación cintura cadera, la circunferencia de la cintura y el pliegue del triceps de los funcionarios del programa de Atención integral de Salud UCR-CCSS.

Según Jakicic (2000) y Haapanen, Miilunpalo Vuori, Oja y Pasanen (1996), las personas deben de generar un gasto calórico mayor de 2000 kilocalorías semanales, porque es en esta cantidad es donde se presentan los mayores beneficios físicos como la resistencia cardiovascular, entre otros.

Ahora bien, los datos que se presentan en la figura 2 muestran que no hay diferencia en cuanto al gasto calórico, ni tampoco en el gasto calórico relativo (figura 3 ), es decir cuando se toma en cuenta el peso de las personas. Contrario a otras investigaciones que señalan que los hombres son más activos que las mujeres (Yang, Telama, Leino y Viikari, 1999; Sternfeld, Ainsworth y Quesenberry, 1999). También este resultado lleva a señalar, que las recomendaciones en cuanto a sexo deberían 
de ser diferentes, porque las mujeres tienen menor peso $(\mathrm{kg})$, por su composición corporal, es decir que poseen menor estatura, por lo que cuentan con menor porcentaje óseo y muscular (Wilmore y Costill, 1994). Entonces para que ellas puedan gastar 2000 kilocalorías, deberían de realizar mayor cantidad de actividad física que los hombres. Esto indica que talvez las recomendaciones para las mujeres deberían de ser inferiores de $2000 \mathrm{kcal}$, o por otro lado las de los hombres mayores a las 2000 kcal, o bien deberían de crearse recomendaciones en cuanto al gasto energético por kilogramo de peso corporal.

En cuanto al gasto calórico de acuerdo a las ocupaciones, se observa en la figura 1 que los de trabajo social, las enfermeras, las (os) secretarias (os), las sociólogas y los mensajeros (as), sí gastan la cantidad adecuada para mantenerse saludables. También se observa que los ATAP son los que gastan más kilocalorías, esto era de esperar porque ellos tienen que caminar durante casi todas las horas de trabajo, ya que visitan a los hogares de la comunidad, para realizar diversas funciones como por ejemplo medir la presión arterial, el peso, realizan encuestas. Curiosamente dentro de los participantes que generan un gasto inferior son los médicos, nutricionistas y auxiliares quienes se dedican a ayudar en las labores de oficina. Ellos no gastan ni 1000 kilocalorías por semana, es decir menos de la mitad de lo recomendado. Este dato es contradictorio en los médicos y nutricionistas, porque se esperaría que ellos fueran activos, ya que además de conocer los beneficios de la práctica regular de actividad física, son profesionales en el área de la salud, por lo que deberían de dar el ejemplo a los pacientes $\mathrm{y}$ ha todas las personas.

Por otro lado cuando se analizan estos resultados en porcentaje, se encuentra que el $40 \%$ de las mujeres son activas, por consiguiente el $60 \%$ restante son mujeres sedentarias, en cuanto a los hombres el 33\% realizan actividad física y el $66,67 \%$ presentan falta de actividad física (tabla 4). Se muestra que estas personas poseen un alto porcentaje de sedentarismo por lo que presentan un factor de riesgo para la salud. Lo que sugiere la puesta en práctica de actividad física, de una forma efectiva, enfatizando en las recomendaciones del Colegio Americano de Medicina Deportiva (Scott, Morrow, Jackson, y Dunn, 2000) estas guías enfatizan la práctica de 30 minutos de actividad física de manera constantes o intermitentes, la mayor cantidad de días por semana, es importante recalcar que la actividad física intermitente es aquella que se realiza de manera fraccionada, por ejemplo 10 minutos constantes 3 veces al día.

En cuanto al IMC (figura 5), las mujeres se encuentran con un IMC de 24.8, lo que indica que se encuentran en el límite superior para clasificarse con sobrepeso, los hombres presentan 25.22 es decir presentan sobrepeso. Por otro lado la figura 6 presenta el porcentaje de la muestra en cada uno de los parámetros establecidos para el IMC, lo que nos índica que el $62 \%$ de la muestra se encuentra con un IMC "idóneo", pero de este $62 \%$, un porcentaje significativo se encuentra con un IMC entre 24-24,9, lo que nos demuestra una cantidad considerable en el límite inferior del sobrepeso, un 23\% de las personas presentan sobrepeso (I.M.C > $24,9<30$ ), y un $14 \%$ se encuentra en obesidad (I.M.C >30). Esto revela que a pesar de ser muy jóvenes (el promedio de edad es de 30 años), poseen altos índices de sobrepeso y obesidad.

Así mismo la obesidad es un factor de riesgo en las enfermedades cardiovasculares (Hernández, 2000; y Esquivel, Suárez, Calzada, Sandí y Ureña, 2001) y si ha esto le sumamos el alto porcentaje de sedentarismo, aumentamos los factores de riesgo por lo que con más razón se deben de tomar cartas en cuanto a la promoción de hábitos saludables en estos funcionarios.

Se va a presentar un ejemplo hipotético: un hombre que pesa $73(\mathrm{~kg})$, con una edad promedio de 30 años, y que su I.M.C. se encuentre en 25 (promedio del I.M.C de los hombres del estudio), sólo tendrían que ganar 120 gramos de peso por mes para que en 10 años se encuentre con obesidad, con 
tan sólo 40 años de edad y además de esto es sedentario, esta persona aumentaría el porcentaje de riesgo. No obstante si a la persona se le educa en cuanto a los estilos de vida saludables podría lograr la adquisición de muchos beneficios tanto físicos como mentales.

Por otra parte en la figura 7 se muestra que los hombres poseen mayor circunferencia de la cintura que las mujeres. Esto es lo esperado porque ellos poseen diámetros más grandes en el cuerpo (Wilmore y Costill, 1994). Un 7.4\% de los hombres tiene una circunferencia superior de 102 centímetros (figura 9), en cuanto a las mujeres un $17.5 \%$ excede a 88 centímetros que es la recomendación para ellas (figura 8), se observa un $14 \%$ de exceso en la circunferencia de la cintura, por lo que nuevamente se señalaría otro aspecto relacionado con las enfermedades cardiovasculares.

Este estudio presenta evidencia en la manifestación de variables que inciden en la salud física de la personas, es imprescindible la intervención de aspectos que modifiquen estos factores de riesgo, como la práctica regular de actividad física, por medio de un plan de acción diseñado de acuerdo a las posibilidades económicas, a las facilidades de acceso a lugares aptos para la práctica física, la contratación de personas especializadas en la educación física, así como también la respectiva educación hacia los funcionarios para que puedan involucrar dentro de la cotidianidad de su vida tanto en el tiempo libre como en el tiempo laboral un estilo de vida más activo.

Para futuras investigaciones se recomienda la utilización de instrumentos que se refieran a aspectos relacionados con la afinidad a la actividad física, así como también los motivos por los cuales no la realizan. Ya que al conocer estas variables, se podrían tomar en cuenta en los planes de acción a seguir, con el objetivo de inculcar la actividad física.

\section{REFERENCIAS}

ACSM (2006). Guidelines for exercise testing and prescription. Seventh Edition. Printer RR Donnellet \& Sons- Crawfordsville. United States of America.

Ainsworth, B., Leon, M., Richardson, D., Jacobs, J. y Paffenbarger (1993). Accuracy of the College Alumnus Physical Activity Questionnaire. Clinical Epidemiological, 46, 1403-1411.

Alemán, C. y Salazar, W. (2003). Niveles de Actividad Física en Estudiantes Universitarios. Investigación sin publicar.

Alemán, C. y Salazar, W. (2004). Niveles de Actividad Física en Funcionarios Públicos. Investigación sin publicar.

Bar- Or, O. (2003). The Juvenile Obesity Epidemic: Is Physical Activity Relevant? Gatorade Sport Science Institute, 16(2), 89.

Bassett, D., Schneider, P. y Huntington, G. (2004). Physical activity in an Old Order Amish Community. Medicine and Science in Sport and Exercise, 36(1), 79-85.

Esquivel, V., Suárez, P., Calzada, L., Sandí, L. y Ureña, J. (2002). Factores de Riesgo Cardiovascular en un grupo de niños escolares obesos Costarricenses. Acta Pediátrica Costarricense. 16 (1), 22-28.

Flegal, K., Carroll, M., Ogden, C. y Jonson, C. (2002). Prevalence and trends in obesity among US adults. JAMA, 288, 1723-1727.

Haapanen, N., Miilunpalo, S., Vuori, L., Oja, P. y Pasanen, M. (1996). Characteristics of Leisure of Premature All- Cause and Cardiovascular Disease Mortality in Middle-aged Men. American Journal of Epidemiology, 143 (9), 870-880.

Hernández, W. (2000). Factores de riesgo de enfermedad cardiovascular en una población obrera industrial de la provincia de Cartago. Revista Costarricense de Salud Publica, 9 (16), 55-64.

Horvat, M., French, R., y Henschen, K (1986). A comparison of the psychological

Jakicic, J., Clark, K., Coleman, E., Donnelly, J., Foreyt, J., Melanson, E., Volek, J. y Volpe, S. (2001). Appropriate Intervention Strategias for Weight Loss and Prevention of Weight Regain for Adults. Medicine and Science in Sports and Exercise, 33(12), 2145-2156.

Katzmarzyk, P (2002). The Cannnadian obesity epidemic: an historical perpective. Obes. Res. 10, 666-674.

Kriska, A.(1997). Introduction to a Collection of Physical Activity Questionnaires. Medicine Science in Sports and Exercise, 29 (6), s5-s9.

Ministerio de Salud (1994). Memoria anual 1993. Departamento de publicaciones e impresos. Costa Rica.189: 19.

Pollock, M.L y Wilmore, J.H. (1990). Exercise in Health and Disease. (2th ed). Philadelphia, PA: W.B. Saunders. 
Powell., K. (1987). Physical activity and the incidence of coronary Herat disease. Annual Review of Public Helth, 8, 253-287.

Pratt, M., Macera, C. y Blanton, C. (1999). Levels of physical activity and inactivity in children and adults in the United States: current evidence and research issues. Medicine and Science in sports and exercise, 31 (11), s526-s533.

Saénz, R (2004). Ministerio de Salud, Sistema Nacional para la prevención y Control de la Obesidad. SEPAN. Datos no publicados.

Sallis, J., Haskell, P. y Wood, P. (1996). Seven- Day Physical Activity Recall. . Medicine Science in Sports and Exercise Supplement, 29 (6), s89-s103.

Sánchez, García, Landabaso y Martínez (1998). Participación e actividad física de una muestra universitaria a partir del modelo de las etapas de cambio en el ejercicio físico un estudio piloto. Revista de Psicología del Deporte, 7 (2), 233-245.

Scott, B., Morrow, J., Jackson, A. y Dunn, A. (2000). Variables related to meeting the CDC/ACSM physical activity guidelines. Medicine and Science in Sports and Exercise, 32(12), 2087-2092.

Serrano, A., Field, J. y Prieto, G. (1994).Obesidad. México: PAIDOS-Pediatriá, 6 (1), 3-12.
Sternfeld, B., Ainsworth, E y Quesenberry, J (1999). Physical activity patterns in diverse -population of women. Preview Medicine, 28, 313-323.

Stewart, G., Trost, N., Owen, N., Sallis, J. y Brown, W. (2002). Correlates of adults'participation in physical activity: review and update. Medicine and Science in Sports Exercise, 34(12), 1996-2001.

Tudor-Locke, C. y Myers, A. (2001). Challenges And Opportunities for Measuring Physical Activity in Sedentary Adults. Sports Medicine, 31 (2), 91-100. Washburn, R., Smith, S., Goldfield, S. y McKinlay, R. (1991). Reliability and physiologic correlates of the Harvard Alumni Activity Survey in a general population. Journal Clinical Epidemiological, 44, 1319-1326.

Wilmore, J. y Costill, D. (1994). Fisiología del Esfuerzo y del Deporte. Editorial Paidotribo, España, 444445.

Yang, X., Telama, R., Leino, M y Viikari, J. (1999). Factors explaining the physical activity of young adults: the importance of early socialization. Scandinavian Journal of Medicine and Science in Sport, 9, 120-127 